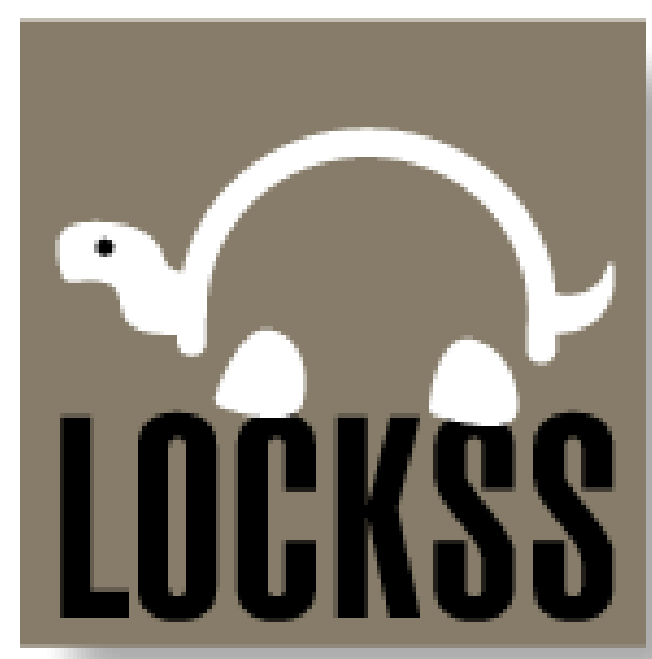

LOTS OF C O P I E S KEE

Victoria Reich

Director LOCKSS Program

Stanford University Libraries

www.lockss.org 


\section{What Is It?}

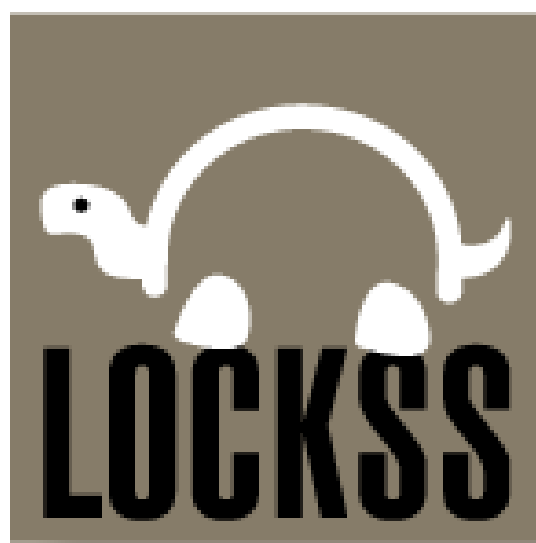

Open Source Peer to Peer

Decentralized

Digital Preservation Infrastructure 
2 ASSUMPTIONS
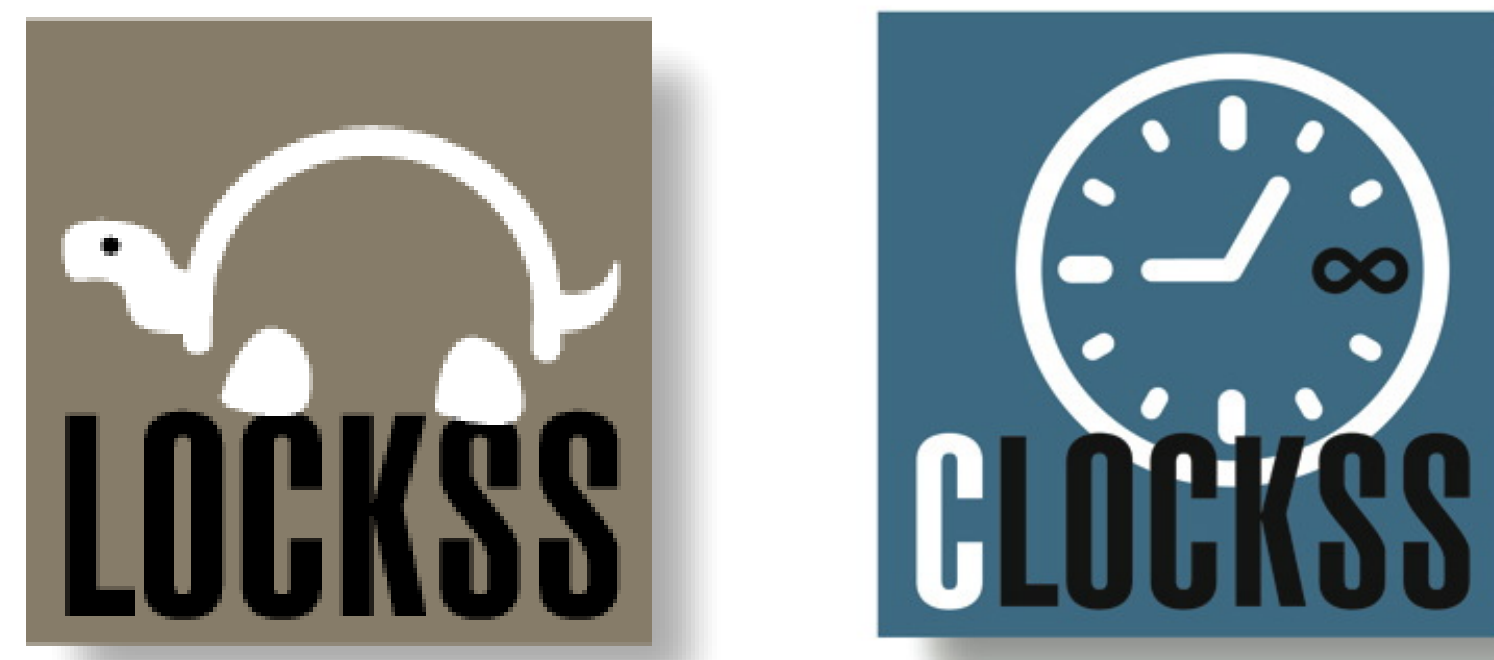


\section{\#1 Democracy -->Libraries}

"Libraries are directly and immediately involved in the conflict which divides our world, and for two reasons. First, because they are essential to the functioning of a democratic society. Second, because the contemporary conflict touches the integrity of scholarship, the freedom of the mind, and even the survival of culture, and libraries are the great tools of scholarship, the great repositories of culture, and the great symbols of the freedom of the mind." $\quad$-Franklin Delano Roosevelt 


\section{\#2 Library -->Collections}

A \{research\} library contains an indepth collection in a particular subject field .... or in-depth collections in several subject fields ...

ALA Glossary 


\section{The Web}

Accidentally changed the business relationship between librarians and publishers.

With rare exceptions, libraries provide access to web materials and do not take physical custody of the content.

The role libraries have played in society for hundreds of years as trusted keepers of information and culture was disrupted.

Our digital cultural and intellectual heritage is at risk. 


\section{Archival Assets}
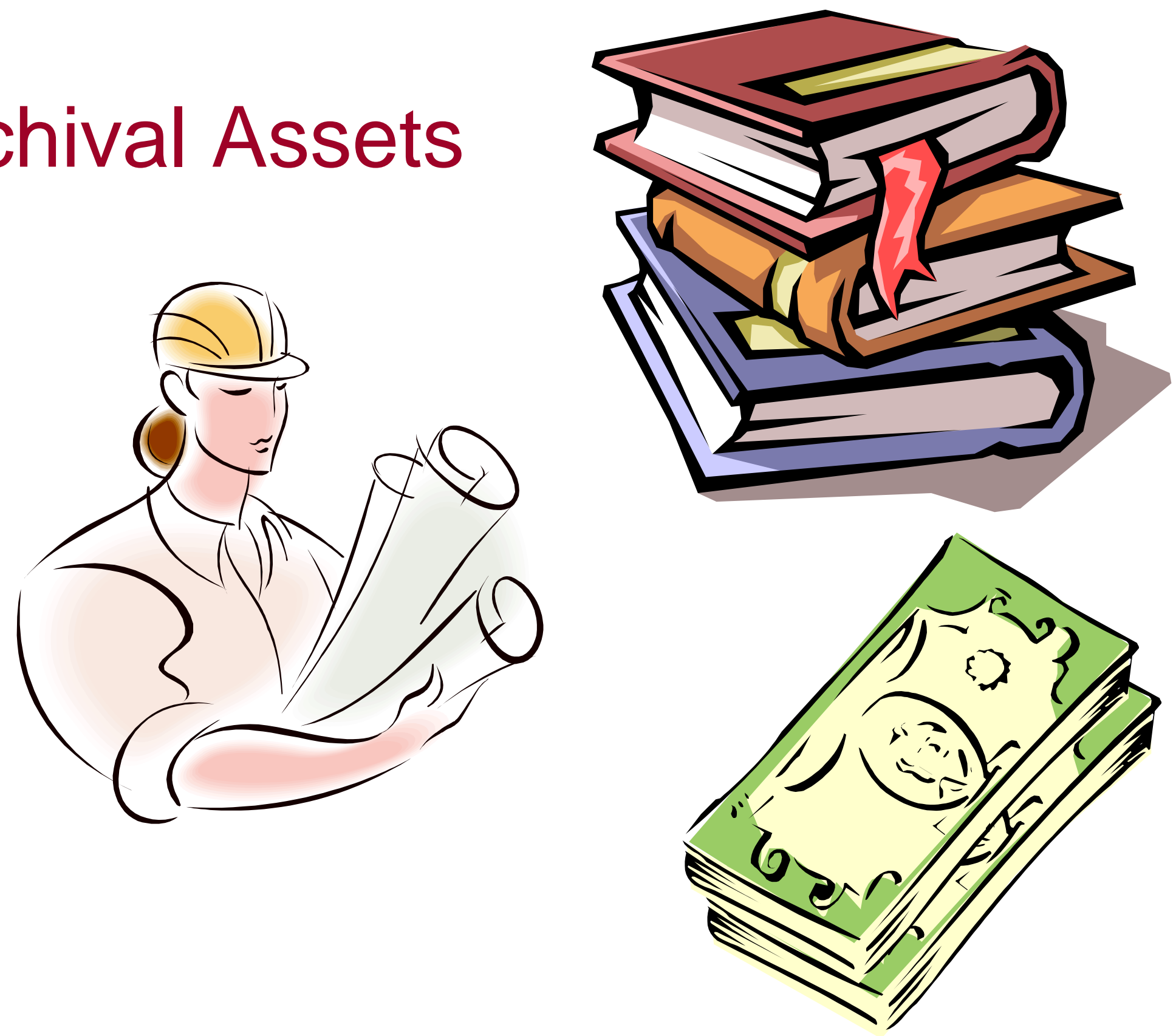


\section{Key Questions}

From this moment on,

- who will have custody

- who will control and govern

societies archival assets? 


\section{Community Governance Community Custody}
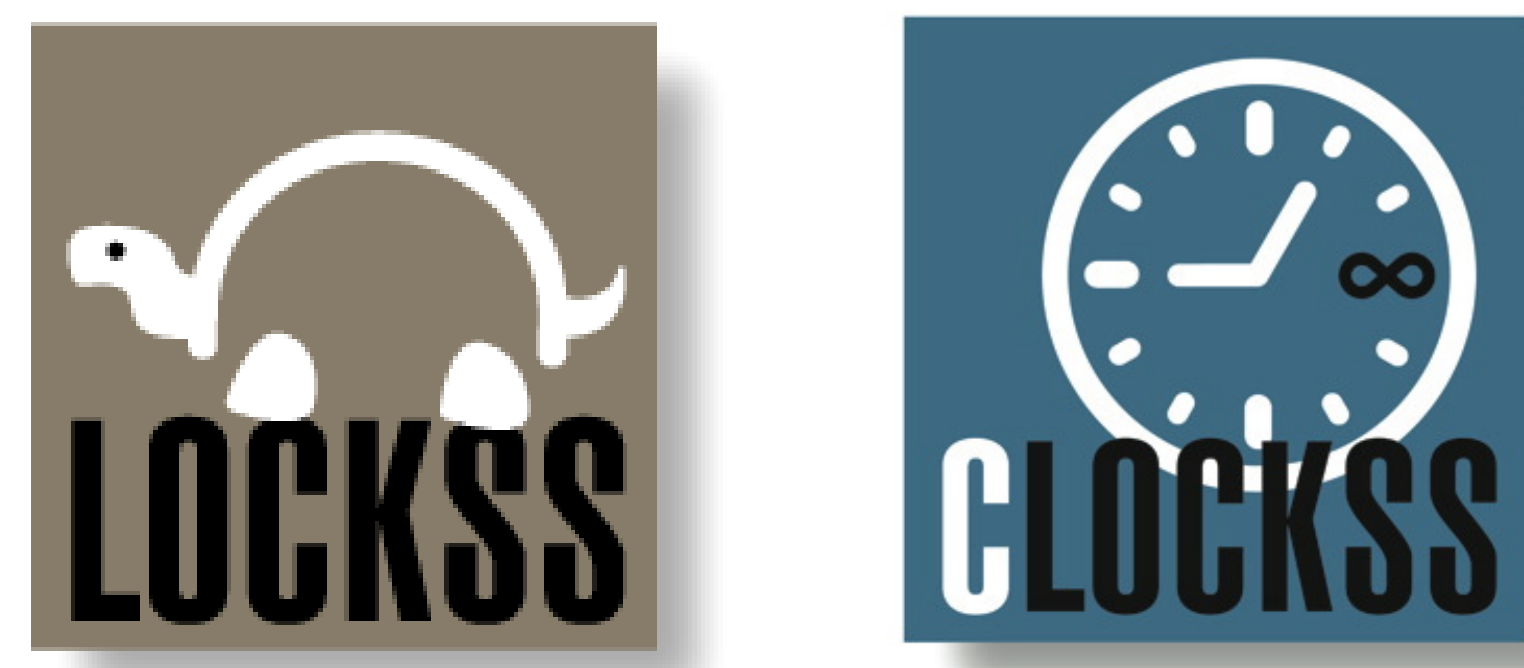


\section{Community Governance Library Custody}

To start: 12 Publishers and 7 libraries Building a distributed, validated, comprehensive archive

Held on behalf of the community

Global access to content post trigger HANDOUT 


\section{Community Governance Library Custody}

Libraries are using LOCKSS to build libraries;

to build \& preserve e-collections

-Subscription materials: eliminating perpetual payment for perpetual access

-Freely available materials: truly and immediately at risk 


\section{The Community Is Keeping LOTS OF STUFF SAFE}

Content that meets these criteria

1. Delivered via the web, http://

2. Has a stable URL structure

3. Has an authoritative version

FORMAT AGNOSTIC -- ALL FILE TYPES 


\section{How Does It Work?}

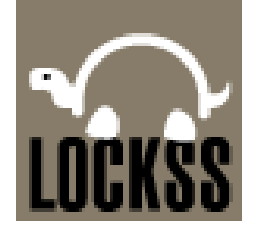




\section{Two Requirements}
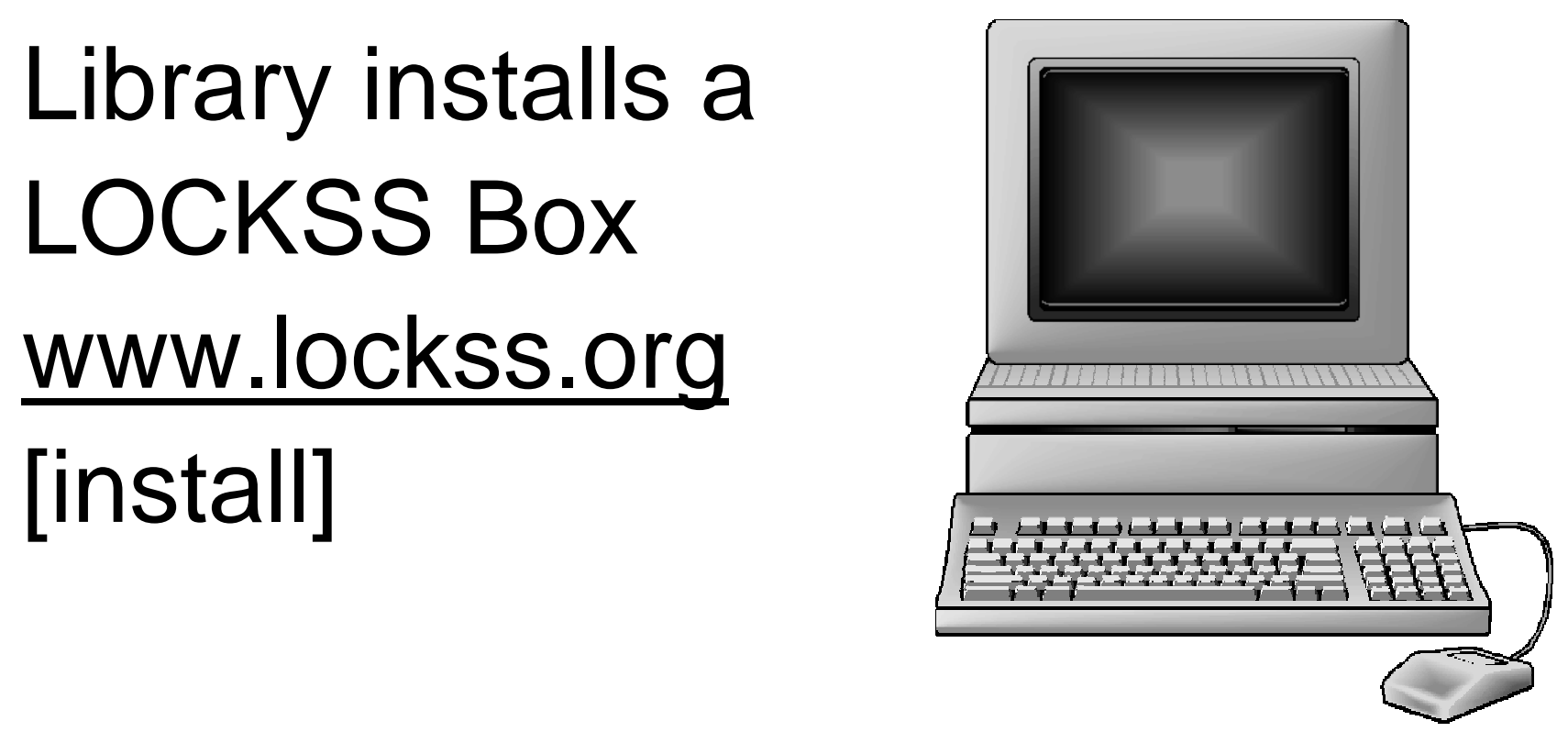

Publisher gives permission 


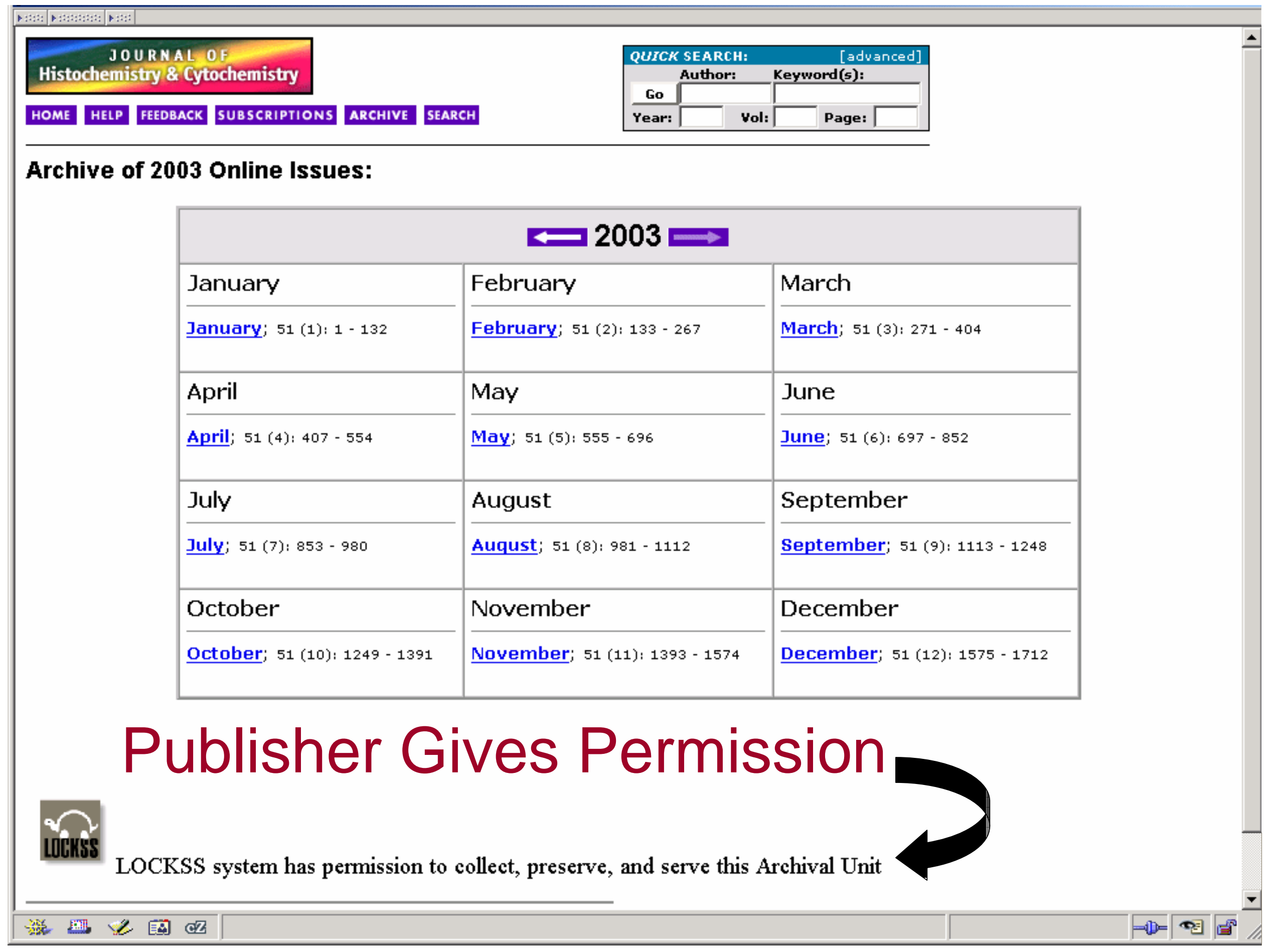




\section{Collection}

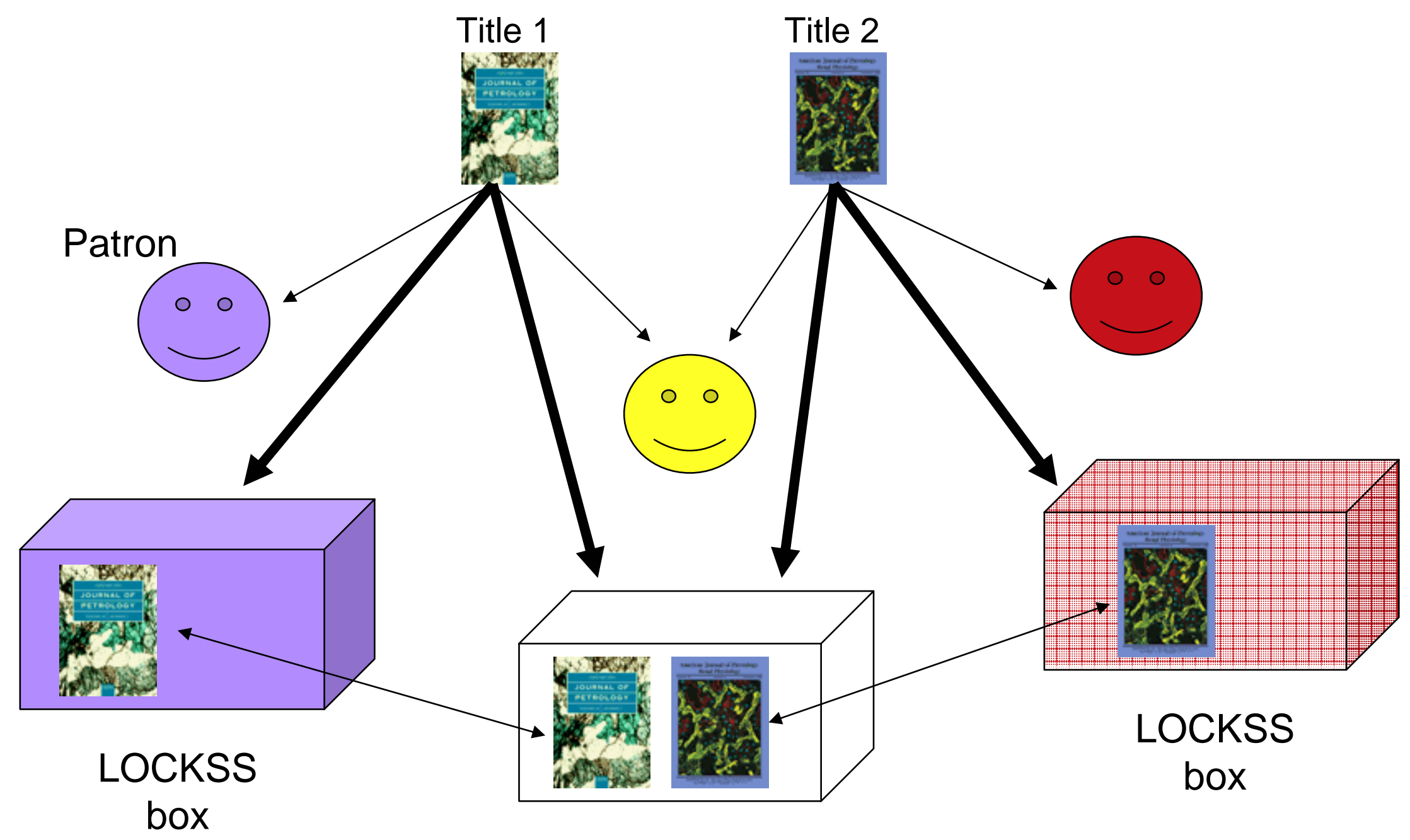

LOCKSS Boxes 


\section{Preservation}

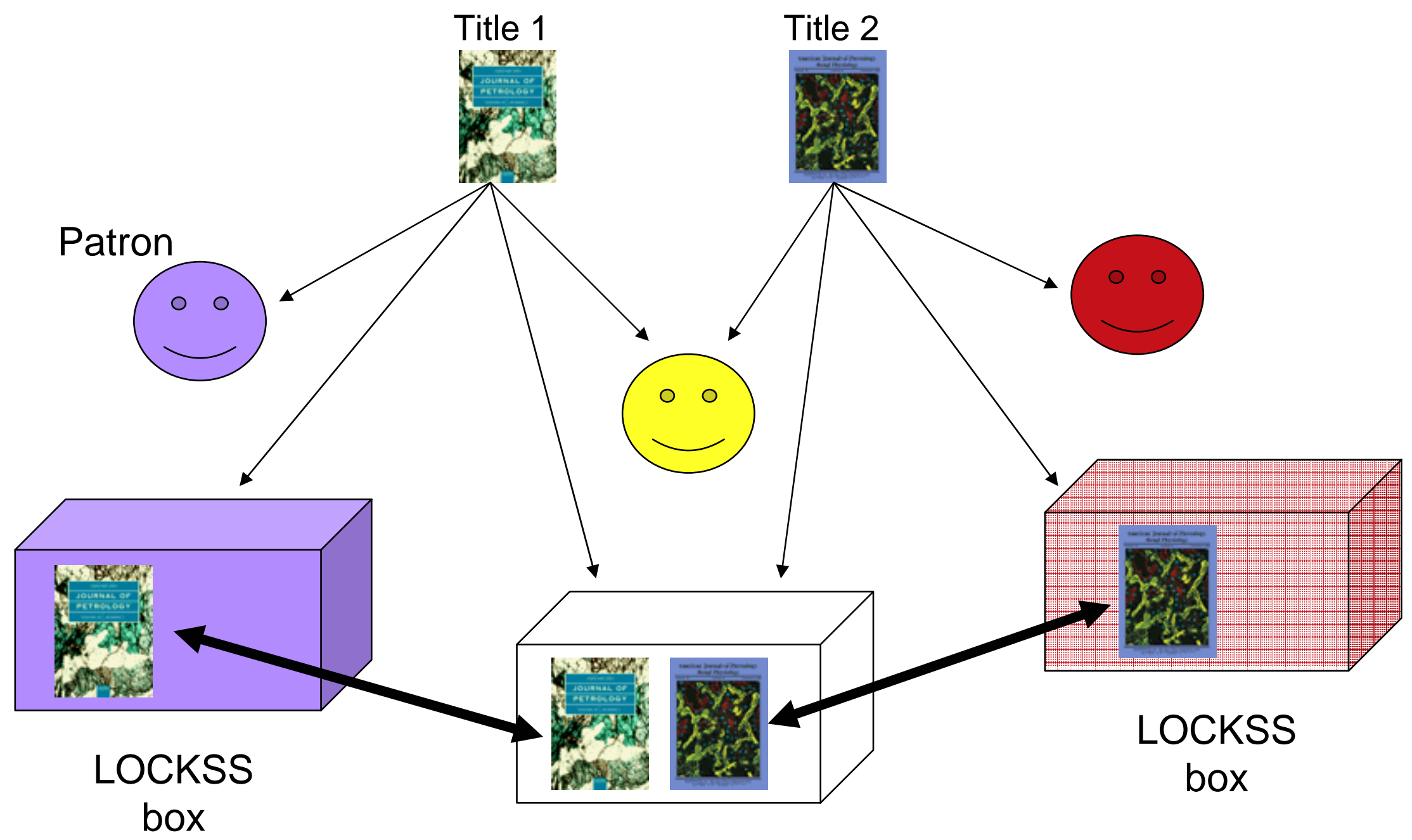

LOCKSS Boxes 


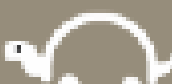 wouks}

\begin{tabular}{|c|c|c|c|c|c|c|c|c|}
\hline $\operatorname{ArC}$ & Units & & & & $\mp$ & & & \\
\hline & Arsh & i linit: & & & & & & \\
\hline Valume & Tantent Siz & $\begin{array}{l}\text { Mink } \\
\text { Whatw } \\
\text { Min }\end{array}$ & Pwrs & Pellls: & Sutus & Inad Pedll & I.wed Cranl & $\begin{array}{l}\text { Inat } \\
\text { Trew Walk }\end{array}$ \\
\hline Ahwirithe Litwran Ranicw son & $1,254,563$ & 71 & Ears & $\frac{13}{\operatorname{pin} \| x}$ & $o t$ & $\begin{array}{l}06:+3: 51 \\
05122 \pi 5\end{array}$ & $\begin{array}{l}06: 40: 53 \\
0512005\end{array}$ & $\begin{array}{l}17: 50: 52 \\
0512205\end{array}$ \\
\hline Amich Tredn Volume sl & $14,5,6,600$ & 16.7 & Dars & $\overline{\frac{36}{\text { Bintis }}}$ & $\omega k$ & $\begin{array}{l}15: 45: 4 \mathrm{~h} \\
0517705\end{array}$ & $\begin{array}{l}23: 51: 54 \\
0512005\end{array}$ & $\begin{array}{l}10: 50: 5 t \\
05127,05\end{array}$ \\
\hline 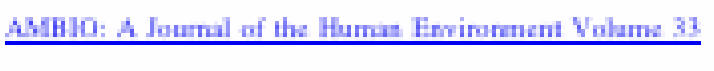 & $79,771,55 \mathrm{~b}$ & $97 \mathrm{E}$ & parrs & $\overline{\frac{19}{p i n}}$ & $\omega k$ & $\begin{array}{l}16: 35: 56 \\
05113.55\end{array}$ & $\begin{array}{l}17+40,04 \\
0512205\end{array}$ & $\begin{array}{l}17: 55: 11 \\
0512705\end{array}$ \\
\hline 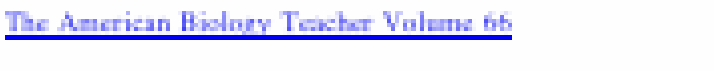 & $40,976,436$ & 59.2 & wars & 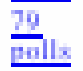 & $\sigma k$ & $\begin{array}{l}09.97: 17 \\
0517305\end{array}$ & $\begin{array}{l}\text { 15:18:39 } \\
\text { 0512005 }\end{array}$ & $\begin{array}{l}01: 15: 19 \\
0512705\end{array}$ \\
\hline Amarican Fum Moumil Volung & $6,76+, 074$ & 13.2 & wars & 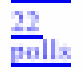 & $\sigma k$ & $\begin{array}{l}\text { 16:5b:09 } \\
\text { 05ituas }\end{array}$ & $\begin{array}{l}\text { 1900:52 } \\
0 \text { iranos }\end{array}$ & $\begin{array}{l}\text { 06: It:04 } \\
0512305\end{array}$ \\
\hline Amarican Hidanizil Resicw Yodume I0E & $21,735,2 \mathrm{kh}$ & 313 & Dars & $\overline{\frac{1}{p i n l l a}}$ & $\sigma k$ & $\begin{array}{l}0901: 39 \\
0412405\end{array}$ & 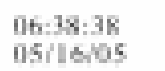 & $\begin{array}{l}01: 55: 43 \\
0512305\end{array}$ \\
\hline Americie llages Volume bo & $4,510,300$ & 12.2 & Bars & $\overline{4}$ & $o k$ & $\begin{array}{l}06: 19: 21 \\
0512305\end{array}$ & $\begin{array}{l}0607.50 \\
05122,55\end{array}$ & $\begin{array}{l}13.55: 37 \\
0512365\end{array}$ \\
\hline Anaricin Jewiah Hetary Walane gi & $17+47,600$ & 24.4 & Barrs & $\overline{\frac{32}{12}}$ & $\omega k$ & $\begin{array}{l}\text { Ohtos:07 } \\
0512705\end{array}$ & $\begin{array}{l}04: 22: 32 \\
0512205\end{array}$ & $\begin{array}{l}\text { Itwo: It } \\
0527,65\end{array}$ \\
\hline Amaricin Jourall of Mathamatios Yolume 125 & $15,207,07 k$ & Inth & Dars & $\overline{\frac{1}{\sin \| x}}$ & $o k$ & $\begin{array}{l}15: 55: 06 \\
0512705\end{array}$ & $\begin{array}{l}12: 31: 24 \\
0512755\end{array}$ & $\begin{array}{l}12: 20: 22 \\
0512705\end{array}$ \\
\hline 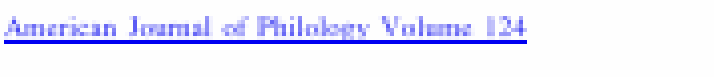 & $11,374,476$ & 17.7 & Bars & $\frac{2 \pi}{\sin \| x}$ & $\sigma k$ & $\begin{array}{l}10: 18: 3 \mathrm{R} \\
0512205\end{array}$ & $\begin{array}{l}00: 90: 39 \\
05122 / 55\end{array}$ & $\begin{array}{l}11+02: 53 \\
05123,05\end{array}$ \\
\hline The Amarian Midland Naturaliat Yolume 152 & $9,177,56 \mathrm{k}$ & 16.6 & Bars: & $\overline{\mathrm{Bin}}$ & $o k$ & $\begin{array}{l}01: 52: 54 \\
0512705\end{array}$ & $\begin{array}{l}20: 44: 41 \\
0512005\end{array}$ & $\begin{array}{l}07: 21: 46 \\
0512105\end{array}$ \\
\hline Anarican Museam Mavilates Wrilume 340 & 943463 & 2.5 & Bars & $\overline{\frac{31}{\operatorname{Bin} \| x}}$ & $o k$ & $\begin{array}{l}06: 3104 \\
05123015\end{array}$ & $\begin{array}{l}21: 35: 0 k \\
05121,45\end{array}$ & $\begin{array}{l}06: 17.52 \\
0512145\end{array}$ \\
\hline Amarizie Guarterly Volune s5 & $10,364,14 \pi$ & 19.6 & Bars & $\overline{\frac{1}{\text { pinlls. }}}$ & $\phi k$ & $\begin{array}{l}17: 48: 41 \\
0512205\end{array}$ & $\begin{array}{l}06: 33: 98 \\
0512205\end{array}$ & $\begin{array}{l}093: 2 \mathrm{k} \\
05 n \mathrm{n}, 5\end{array}$ \\
\hline 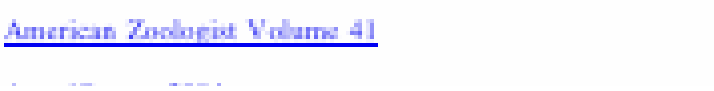 & $51,675,406$ & 80.3 & Bars & in & $\sigma k$ & $\begin{array}{l}23: 17: 16 \\
05120155\end{array}$ & $\begin{array}{l}14: 16: 17 \\
05113,05\end{array}$ & $\begin{array}{l}21.39: 47 \\
0512305\end{array}$ \\
\hline
\end{tabular}

Daemon Status

\section{Archival Units}

Arshioal linit.
Imenal Corienation

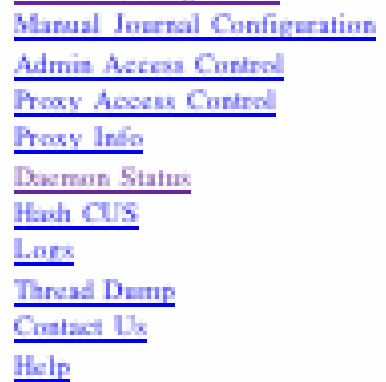

Halp

\begin{tabular}{|c|c|c|c|c|c|c|c|c|}
\hline $\operatorname{ArC}$ & Units & & & & $\mp$ & & & \\
\hline & Arsh & i linit: & & & & & & \\
\hline Valume & Tantent Siz & $\begin{array}{l}\text { Mink } \\
\text { Whatw } \\
\text { Min }\end{array}$ & Pwrs & Pellls: & Sutus & Inad Pedll & I.wed Cranl & $\begin{array}{l}\text { Inat } \\
\text { Trew Walk }\end{array}$ \\
\hline Ahwirithe Litwran Ranicw son & $1,254,563$ & 71 & Ears & $\frac{13}{\operatorname{pin} \| x}$ & $o t$ & $\begin{array}{l}06:+3: 51 \\
05122 \pi 5\end{array}$ & $\begin{array}{l}06: 40: 53 \\
0512005\end{array}$ & $\begin{array}{l}17: 50: 52 \\
0512205\end{array}$ \\
\hline Amich Tredn Volume sl & $14,5,6,600$ & 16.7 & Dars & $\overline{\frac{36}{\text { Bintis }}}$ & $\omega k$ & $\begin{array}{l}15: 45: 4 \mathrm{~h} \\
0517705\end{array}$ & $\begin{array}{l}23: 51: 54 \\
0512005\end{array}$ & $\begin{array}{l}10: 50: 5 t \\
05127,05\end{array}$ \\
\hline 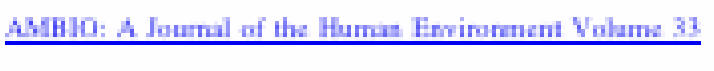 & $79,771,55 \mathrm{~b}$ & $97 \mathrm{E}$ & parrs & $\overline{\frac{19}{p i n}}$ & $\omega k$ & $\begin{array}{l}16: 35: 56 \\
05113.55\end{array}$ & $\begin{array}{l}17+40,04 \\
0512205\end{array}$ & $\begin{array}{l}17: 55: 11 \\
0512705\end{array}$ \\
\hline 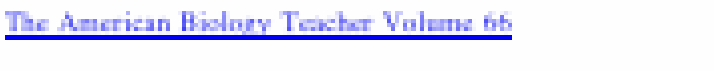 & $40,976,436$ & 59.2 & wars & 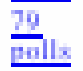 & $\sigma k$ & $\begin{array}{l}09.97: 17 \\
0517305\end{array}$ & $\begin{array}{l}\text { 15:18:39 } \\
\text { 0512005 }\end{array}$ & $\begin{array}{l}01: 15: 19 \\
0512705\end{array}$ \\
\hline Amarican Fum Moumil Volung & $6,76+, 074$ & 13.2 & wars & 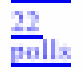 & $\sigma k$ & $\begin{array}{l}\text { 16:5b:09 } \\
\text { 05ituas }\end{array}$ & $\begin{array}{l}\text { 1900:52 } \\
0 \text { iranos }\end{array}$ & $\begin{array}{l}\text { 06: It:04 } \\
0512305\end{array}$ \\
\hline Amarican Hidanizil Resicw Yodume I0E & $21,735,2 \mathrm{kh}$ & 313 & Dars & $\overline{\frac{1}{p i n l l a}}$ & $\sigma k$ & $\begin{array}{l}0901: 39 \\
0412405\end{array}$ & 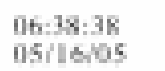 & $\begin{array}{l}01: 55: 43 \\
0512305\end{array}$ \\
\hline Americie llages Volume bo & $4,510,300$ & 12.2 & Bars & $\overline{4}$ & $o k$ & $\begin{array}{l}06: 19: 21 \\
0512305\end{array}$ & $\begin{array}{l}0607.50 \\
05122,55\end{array}$ & $\begin{array}{l}13.55: 37 \\
0512365\end{array}$ \\
\hline Anaricin Jewiah Hetary Walane gi & $17+47,600$ & 24.4 & Barrs & $\overline{\frac{32}{12}}$ & $\omega k$ & $\begin{array}{l}\text { Ohtos:07 } \\
0512705\end{array}$ & $\begin{array}{l}04: 22: 32 \\
0512205\end{array}$ & $\begin{array}{l}\text { Itwo: It } \\
0527,65\end{array}$ \\
\hline Amaricin Jourall of Mathamatios Yolume 125 & $15,207,07 k$ & Inth & Dars & $\overline{\frac{1}{\sin \| x}}$ & $o k$ & $\begin{array}{l}15: 55: 06 \\
0512705\end{array}$ & $\begin{array}{l}12: 31: 24 \\
0512755\end{array}$ & $\begin{array}{l}12: 20: 22 \\
0512705\end{array}$ \\
\hline 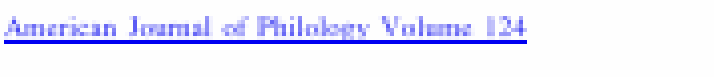 & $11,374,476$ & 17.7 & Bars & $\frac{2 \pi}{\sin \| x}$ & $\sigma k$ & $\begin{array}{l}10: 18: 3 \mathrm{R} \\
0512205\end{array}$ & $\begin{array}{l}00: 90: 39 \\
05122 / 55\end{array}$ & $\begin{array}{l}11+02: 53 \\
05123,05\end{array}$ \\
\hline The Amarian Midland Naturaliat Yolume 152 & $9,177,56 \mathrm{k}$ & 16.6 & Bars: & $\overline{\mathrm{Bin}}$ & $o k$ & $\begin{array}{l}01: 52: 54 \\
0512705\end{array}$ & $\begin{array}{l}20: 44: 41 \\
0512005\end{array}$ & $\begin{array}{l}07: 21: 46 \\
0512105\end{array}$ \\
\hline Anarican Museam Mavilates Wrilume 340 & 943463 & 2.5 & Bars & $\overline{\frac{31}{\operatorname{Bin} \| x}}$ & $o k$ & $\begin{array}{l}06: 3104 \\
05123015\end{array}$ & $\begin{array}{l}21: 35: 0 k \\
05121,45\end{array}$ & $\begin{array}{l}06: 17.52 \\
0512145\end{array}$ \\
\hline Amarizie Guarterly Volune s5 & $10,364,14 \pi$ & 19.6 & Bars & $\overline{\frac{1}{\text { pinlls. }}}$ & $\phi k$ & $\begin{array}{l}17: 48: 41 \\
0512205\end{array}$ & $\begin{array}{l}06: 33: 98 \\
0512205\end{array}$ & $\begin{array}{l}093: 2 \mathrm{k} \\
05 n \mathrm{n}, 5\end{array}$ \\
\hline 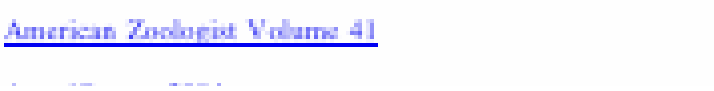 & $51,675,406$ & 80.3 & Bars & in & $\sigma k$ & $\begin{array}{l}23: 17: 16 \\
05120155\end{array}$ & $\begin{array}{l}14: 16: 17 \\
05113,05\end{array}$ & $\begin{array}{l}21.39: 47 \\
0512305\end{array}$ \\
\hline
\end{tabular}




\section{Access}

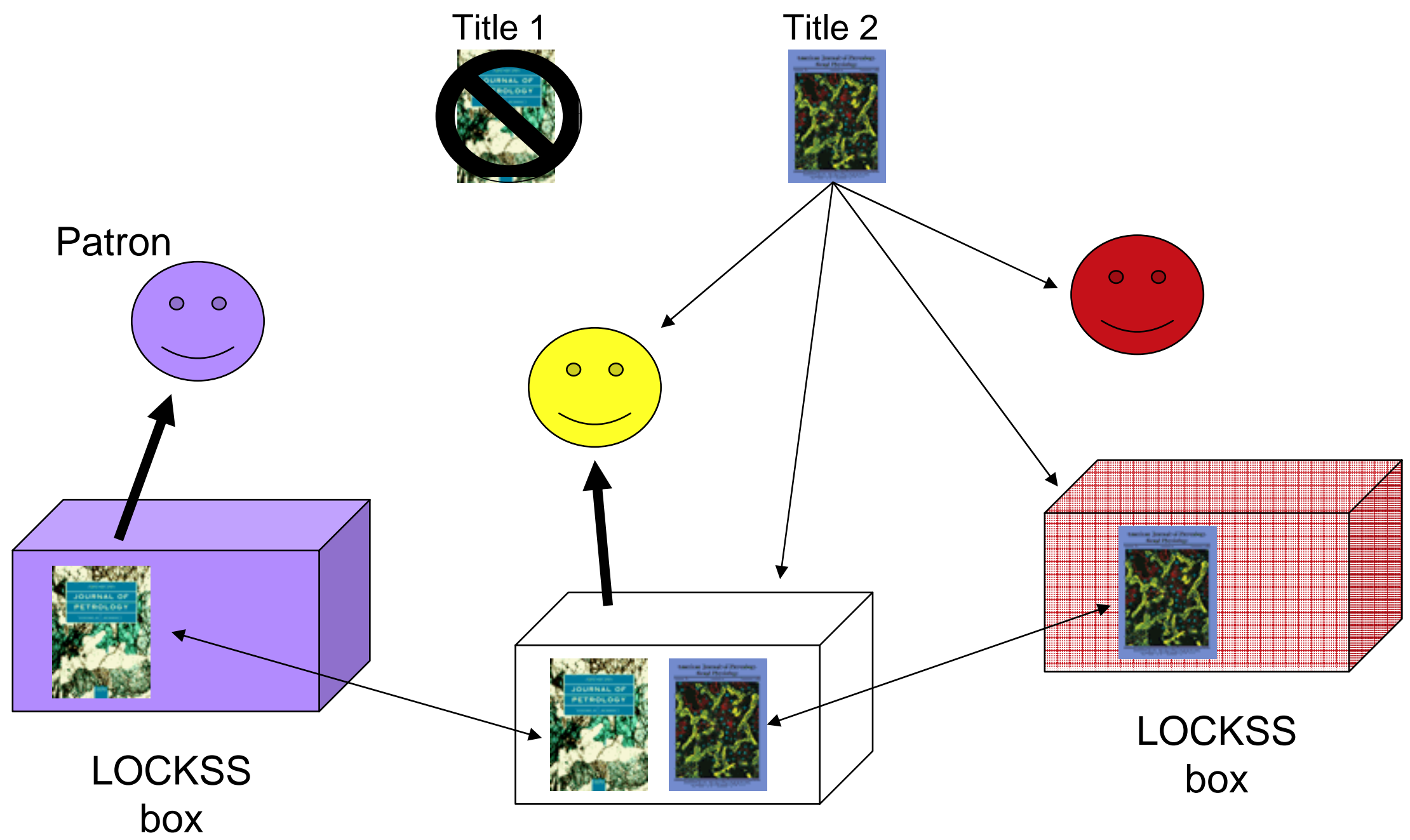

Prevents the publisher from revoking access rights to back content 


\section{Look and Feel to Readers}

When content is served to the user from a LOCKSS Box

- Look and feel is as close as possible to what the publisher published

-Preserve content \& presentation 


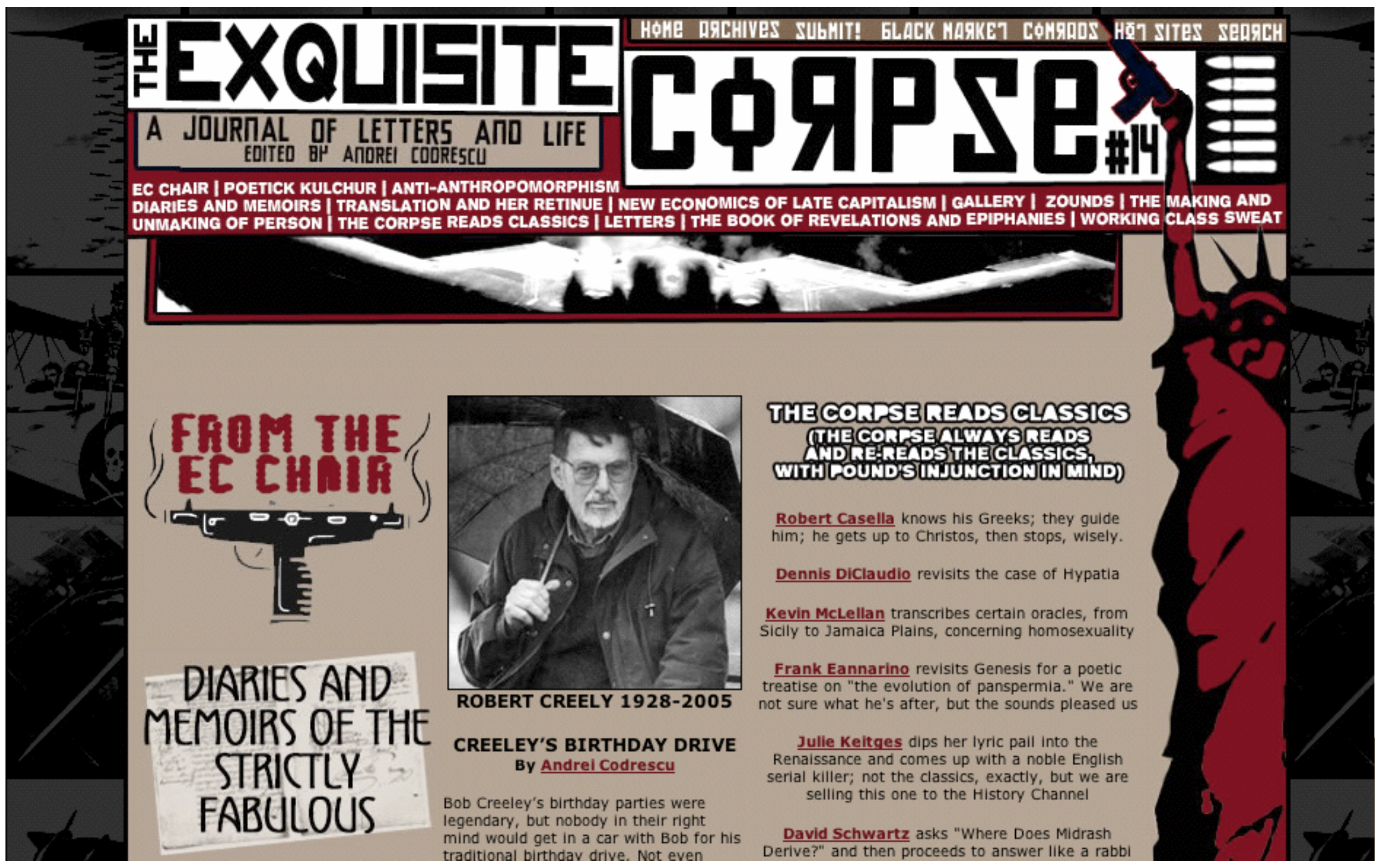




\section{Format Migration}

"on the fly"

When content is requested

Process is transparent to the reader

D-Lib Magazine, Volume 11, Number 1, January, 2005

http://www.dlib.org/dlib/january05/

rosenthal/01rosenthal.html 


\section{Format Migration "on the fly"}

Preserve the original look \& feel. Value in increasing amounts of content.

Reduce the cost of ingest. Preserve more material per dollar.

Postpone costs of migration. Leverage time value of money \& technology cost curve.

Migrate material upon reader request.

Vastly lower the amount of content processed.

What the readers sees is the result of

the best possible technology at time of access. 
THELBRARY COONGRESS

\section{NDIIPP}

Audit: Threats Models

Audit: Bit Preservation 


\section{Audit \\ Threat Models}

2005 National Science Board report to

NARA

......the designers of a digital preservation

system need a clear vision of the

threats against which they are being asked to protect their system's contents, and those threats under which it is acceptable for preservation to fail. 


\section{Threats}

Continuous and Abrupt changes

Technology

- storage media, hardware, software, formats

Money

- here one day, gone the next

Organizations

- shifting priorities, politics, staffs

Natural disasters

Human errors and attacks 


\section{Biggest threat to digital preservation is money}

Digital bits are dynamic.

If you don't preserve them you loose them.

Digital preservation has to be a "marginal expense"; you can't "halt" the preservation process and continue to have collections. 


\section{Bit Preservation}

Digital bits are dynamic

- Must be preserved on a dynamic system

Must have lots of copies

- Copies must be loosely coupled

- Don't make Winston Smith's job easy 


\section{Audit \\ Bit Preservation}

Develop low-level system protocols and standards

Build on peer to peer LOCKSS audit and repair protocol

Build on draft LC bit preservation functional requirements 


\section{Join Us}
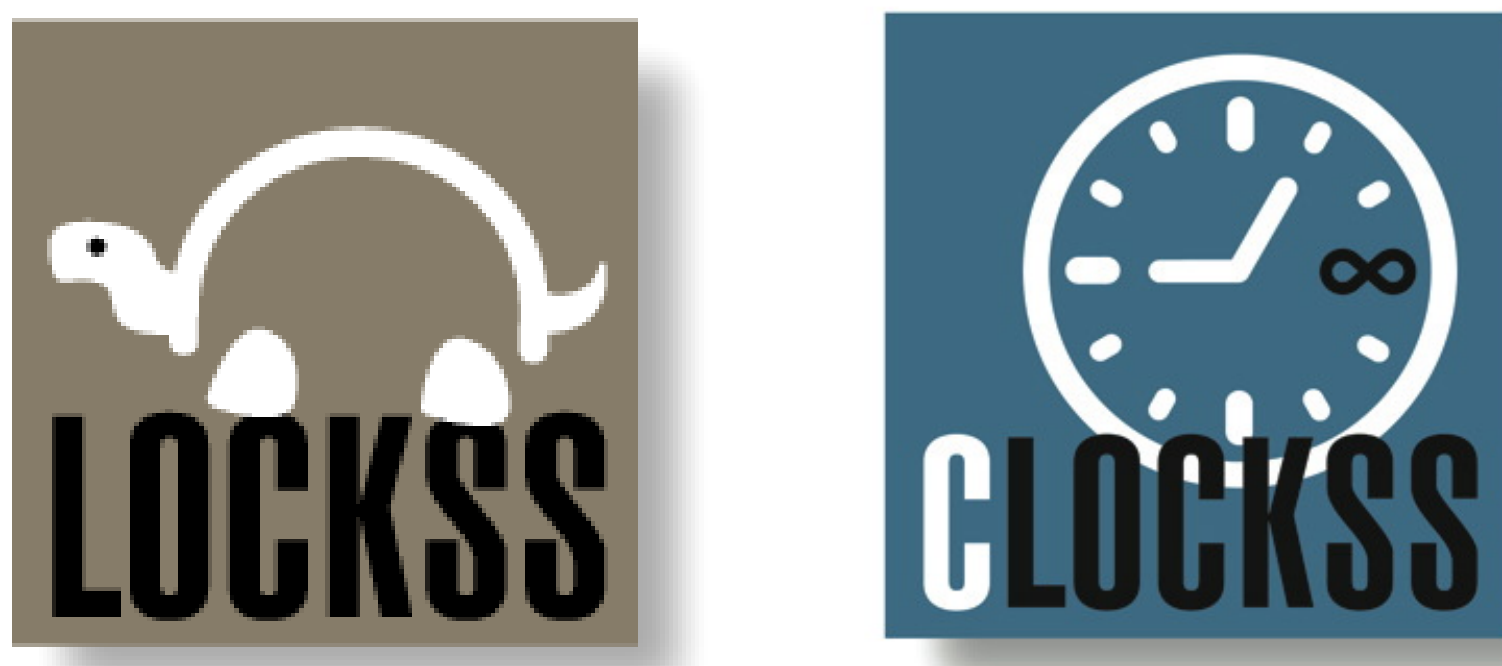